\title{
Do static sources outside a Schwarzschild black hole radiate?
}

\author{
Atsushi Higuchi \\ Institut für theoretische Physik, Universität Bern, Sidlerstrasse 5, CH-3012 Bern, Switzerland \\ and Department of Mathematics, University of York, Heslington, York YO1 5DD, United Kingdom \\ George E. A. Matsas \\ Instituto de Física Teórica, Universidade Estadual Paulista, Rua Pamplona 145, 01405-900-São Paulo, São Paulo, Brazil \\ Daniel Sudarsky \\ Instituto de Ciencias Nucleares, Universidad Nacional Autónoma de México, A. Postal 70/543, México D.F. 04510, Mexico
}

(Received 25 November 1996)

\begin{abstract}
We show that static sources coupled to a massless scalar field in Schwarzschild spacetime give rise to emission and absorption of zero-energy particles due to the presence of Hawking radiation. This is in complete analogy with the description of the bremsstrahlung by a uniformly accelerated charge from the coaccelerated observers' point of view. The response rate of the source is found to coincide with that in Minkowski spacetime as a function of its proper acceleration. It is interesting that this quantum result appears to reflect the classical equivalence principle. [S0556-2821(97)50122-6]
\end{abstract}

PACS number(s): 04.70.Dy, 04.62.+v

The relation between radiation from accelerated charges and the equivalence principle has for some time been the source of much confusion and discussion. A particularly interesting question is how to reconcile the following two facts (in Minkowski spacetime): On the one hand, an accelerated charge is known to radiate when it is seen from the viewpoint of inertial observers. On the other hand, according to the equivalence principle, the same charge is seen by comoving observers as a static charge in a uniform "gravitational field,' ' and, hence, is not expected to radiate. In the classical context, this question has been answered first by Rohrlich [1] and further clarified by Boulware [2], who has shown that the presence of a horizon for the collection of comoving observers, who perceive the charge as static, serves to explain the apparent paradox. This resolution is based on the fact that the radiation zone (as described by the Minkowski observers) lies beyond the comoving observers' horizon and is thus unobservable by them. In the quantum mechanical context, the solution to this paradox (which is now cast in terms of photon emission rates) has been given by the authors [3], by recalling that, as seen by the comoving observers, the static charge (which has in fact constant proper acceleration) is immersed in the Fulling-Davies-Unruh (FDU) thermal bath [4-6] in Rindler spacetime [7]. That is, the interaction of the static charge with this thermal bath results in the absorption and stimulated emission of photons with zero Rindler energy (which are actually nondetectable by the comoving observers), and this completely accounts for the bremsstrahlung due to a uniformly accelerated charge in quantum electrodynamics as described by inertial observers. (Here, the Rindler energy means the energy corresponding to the boost Killing vector field with respect to which Rindler spacetime is static.)

The purpose of this paper is to note that, in complete analogy to the result obtained in the case of the static charge in Rindler spacetime as described before, the analysis of a static source in a static black-hole spacetime, which interacts with the Hawking radiation [8], yields a finite response rate. In fact we will see that the total response rate is exactly the same as that of a uniformly accelerated source in Minkowski spacetime as a function of the proper acceleration.

It should be noted that our source is a classical test source, which influences the scalar field, but is not influenced by it. This point should be emphasized because there is a controversy as to whether or not a uniformly accelerated UnruhDeWitt detector [5,9], which fully interacts with the field, radiates and, if so, how it radiates [10-15]. Although we are considering classical sources, accelerated detectors are similar to them in some respects. We favor the viewpoint that a classical accelerated source radiates at a constant rate because a constant classical Minkowski energy flux is present far away from the source [16]. Nevertheless, it is often asserted that the particles emitted by the accelerated classical source are radiated only when the acceleration is changed. Although we do not share this viewpoint, it will not be in conflict with our results if the word "response rate" is replaced by "time-averaged response rate." We would also like to point out that our main result can still be taken as the fact that the integrated two-point function for a free massless scalar field $\Phi(x), \int d \tau\langle\Phi[x(\tau)] \Phi[x(0)]\rangle$, where $x^{\mu}(\tau)$ is the static world line in Schwarzschild spacetime and $\tau$ is the proper time, coincides with the corresponding quantity in Rindler spacetime as a function of the acceleration.

We first review the general formalism for computing the response rate of a classical source in a static spacetime and the result of Ref. [3] in the context of massless scalar field [17]. Then we present our result for Schwarzschild spacetime.

Let us consider a globally hyperbolic static spacetime described by the metric

$$
d s^{2}=f(\mathbf{x}) d t^{2}-h_{i j}(\mathbf{x}) d x^{i} d x^{j}
$$


We will study a real scalar field $\Phi$ that interacts with a classical source $j(x)[x=(t, \mathbf{x})]$ and is described by the action

$$
S=\int d^{4} x \sqrt{f h}\left(\frac{1}{2} \nabla^{\mu} \Phi \nabla_{\mu} \Phi+j \Phi\right),
$$

where $h(\mathbf{x})=\operatorname{det} h_{i j}(\mathbf{x})$. Let

$$
u_{\omega \mathrm{s} \lambda}(x)=\sqrt{\frac{\omega}{\pi}} U_{\omega \mathrm{s} \lambda}(\mathbf{x}) \exp (-i \omega t)
$$

with $\omega>0$ and their complex conjugates, $u_{\omega \mathrm{s} \lambda}(x)^{*}$, be solutions to $\square u=0$, where $\mathbf{s}=\left(s_{1}, \cdots, s_{n}\right)$ is a set of continuous labels and $\lambda$ is a discrete label for the complete set of modes. We have assumed $\omega$ to be continuous because this is the case in the spacetimes we study, and adopted it as one of the labels. The factor of $\sqrt{\omega / \pi}$ has been inserted for later convenience. Let these solutions be Klein-Gordon orthonormalized:

$$
\begin{gathered}
i \int d \Sigma n^{\mu}\left(u_{\omega \mathrm{s} \lambda}^{*} \nabla_{\mu} u_{\omega^{\prime} \mathbf{s}^{\prime} \lambda^{\prime}}-\nabla_{\mu} u_{\omega \mathrm{s} \lambda}^{*} \cdot u_{\omega^{\prime} \mathbf{s}^{\prime} \lambda^{\prime}}\right) \\
=\delta\left(\omega-\omega^{\prime}\right) \delta\left(\mathbf{s}-\mathbf{s}^{\prime}\right) \delta_{\lambda \lambda^{\prime}}, \\
i \int d \Sigma n^{\mu}\left(u_{\omega \mathrm{s} \lambda} \nabla_{\mu} u_{\omega^{\prime} \mathbf{s}^{\prime} \lambda^{\prime}}-\nabla_{\mu} u_{\omega \mathrm{s} \lambda} \cdot u_{\omega^{\prime} \mathbf{s}^{\prime} \lambda^{\prime}}\right)=0,
\end{gathered}
$$

where $d \Sigma$ is the volume element of a Cauchy surface and where $n^{\mu}$ is the future-pointing unit normal to it. The in-field $\Phi^{\text {in }}$ satisfying the free field equation $\square \Phi^{\text {in }}=0$ can now be expanded as

$$
\Phi^{\mathrm{in}}(x)=\sum_{\lambda} \int d \omega d^{n} \mathbf{s}\left[u_{\omega \mathrm{s} \lambda}(x) a_{\omega \mathrm{s} \lambda}^{\mathrm{in}}+\text { H.c. }\right] .
$$

Let the initial state be the in-vacuum state $|0\rangle_{\text {in }}$ defined by $a_{\omega \mathbf{s} \lambda}^{\text {in }}|0\rangle_{\text {in }}=0$ for all $\omega, \mathbf{s}$, and $\lambda$.

We will be interested in static sources. However, as we will see later, we need to introduce oscillation as a regulator in order to avoid the appearance of intermediate indefinite results. Therefore we consider at this point a source of the form

$$
j_{\omega_{0}}(x)=J(\mathbf{x}) \cos \omega_{0} t
$$

but eventually we make $\omega_{0} \rightarrow 0$. The rate of spontaneous emission with fixed $\mathbf{s}$ and $\lambda$ can now be found to lowest order in perturbation theory:

$$
R_{\mathrm{sp}}\left(\omega_{0} ; \mathbf{s}, \lambda\right) d^{n} \mathbf{s}=\frac{\omega_{0}}{2}\left|\widetilde{J}\left(\omega_{0}, \mathbf{s}, \lambda\right)\right|^{2} d^{n} \mathbf{s}
$$

where

$$
\widetilde{J}\left(\omega_{0}, \mathbf{s}, \lambda\right)=\int d^{3} \mathbf{x} \sqrt{h(\mathbf{x}) f(\mathbf{x})} J(\mathbf{x}) U_{\omega_{0} \mathbf{s} \lambda}(\mathbf{x}) .
$$

We note that Eq. (4) gives the emission rate per unit coordinate time. Later we will convert it into the rate per unit proper time for point sources.
If the source is immersed in a thermal bath of inverse temperature $\beta=1 / k_{B} T$, the rates of absorption and induced emission are both $R_{\mathrm{sp}}\left(\omega_{0} ; \mathbf{s}, \lambda\right) /\left(\exp \beta \omega_{0}-1\right)$. Summing the absorption rate and the spontaneous and induced emission rates, we find the total response rate:

$$
R\left(\omega_{0} ; \mathbf{s}, \lambda\right)=\frac{\omega_{0}}{2} \operatorname{coth} \frac{\beta \omega_{0}}{2}\left|\widetilde{J}\left(\omega_{0}, \mathbf{s}, \lambda\right)\right|^{2} .
$$

In the case of interest here, i.e., for $\omega_{0} \rightarrow 0$, we have

$$
R(0 ; \mathbf{s}, \lambda)=\beta^{-1}|\widetilde{J}(0, \mathbf{s}, \lambda)|^{2} .
$$

This is a useful intermediate result: The rate of zero-energy particles with quantum numbers $(\mathbf{s}, \lambda)$ emitted and absorbed by a static scalar source immersed in a thermal bath at the temperature $\beta^{-1}$ is given by Eq. (5).

Let us now review how the bremsstrahlung rate due to a uniformly accelerated source in Minkowski spacetime (covered with inertial observers) is reproduced in Rindler spacetime (covered with uniformly accelerated observers) by taking the FDU thermal bath into account. First we present the conventional result for the emission rate, which is to be compared with the Rindler-spacetime result. We define the Rindler coordinates $\tau$ and $\xi$ in terms of the usual Minkowski coordinates by

$$
t=a^{-1} e^{a \xi} \sinh a \tau, \quad z=a^{-1} e^{a \xi} \cosh a \tau,
$$

with which we express our classical source as

$$
j_{0}=q \delta(\xi) \delta(x) \delta(y) .
$$

This source has constant proper acceleration $a$. Using the standard method (see, e.g., Ref. [18]), we obtain the rate of spontaneous emission of particles with fixed transverse momentum $\left(k_{x}, k_{y}\right)$ :

$$
\begin{aligned}
R_{\mathrm{sp}}^{M}\left(k_{x}, k_{y}\right) d k_{x} d k_{y} & =q^{2} \int_{-\infty}^{+\infty} d w \Delta_{k_{\perp}}\left(\frac{2}{a}\left|\sinh \frac{a w}{2}\right|\right) \frac{d k_{x} d k_{y}}{(2 \pi)^{2}} \\
& =\frac{q^{2}}{4 \pi^{3} a}\left[K_{0}\left(k_{\perp} / a\right)\right]^{2} d k_{x} d k_{y}
\end{aligned}
$$

where $k_{\perp}=\sqrt{k_{x}^{2}+k_{y}^{2}}$. (We refer the reader to Ref. [19] for formulas involving special functions used in this paper.) The function $\Delta_{m}(\sqrt{\sigma})=-\frac{1}{4} N_{0}(m \sqrt{\sigma})$, where $\sigma=t^{2}-z^{2}$, is the symmetrized two-point function of massive scalar field in two dimensions with $\sigma>0$.

We can now compare the rate (6) with the rate obtained by uniformly accelerated observers in Rindler spacetime:

$$
d s^{2}=e^{2 a \xi}\left(d \tau^{2}-d \xi^{2}\right)-d x^{2}-d y^{2} .
$$

Note that variable $\tau$ is adopted as time. We first note that from this perspective the source is immersed in the FDU thermal bath. This source absorbs particles from the heat bath, which also gives rise to induced emission. Since the particle concept depends on the timelike Killing vector that one uses to define it, the emission of a Minkowski particle (i.e., one defined with respect to $\partial / \partial t$ ) can correspond either 
to absorption or to emission of a Rindler particle (i.e., one defined with respect to $\partial / \partial \tau$ ) [20]. However, the rate of $r e$ sponse, i.e., emission plus absorption, must be independent of the description that one uses. Therefore, the rate of spontaneous emission given by Eq. (6) should equal the total response rate of the source $j_{0}$ computed in Rindler spacetime with the FDU thermal bath.

There is a technical complication with the verification of the above statement due to the fact that the spontaneous emission rate vanishes because the source is now static whereas the density of states in the thermal bath diverges in the zero-frequency limit. As a result, we encounter an expression of the form $0 \times \infty$ in the process of computing the response rate using the particle concept in Rindler spacetime. For this reason we regularize the calculation by considering

$$
j=\sqrt{2} q \cos \omega_{0} \tau \delta(\xi) \delta(x) \delta(y)
$$

and taking the limit $\omega_{0} \rightarrow 0$ in the end. The factor of $\sqrt{2}$ is necessary to make the time average of the squared charge equal $q^{2}$. The source (7) is then equivalent to the source $j_{0}$ in the limit $\omega_{0} \rightarrow 0$ because the rate is proportional to the squared charge at the lowest order.

Now we verify explicitly that the $\omega_{0} \rightarrow 0$ limit of the total response rate of the source (7), which is obtained from Eq. (5) with $\beta^{-1}=a / 2 \pi$, coincides with the rate (6). The positive-frequency modes with respect to $i \partial / \partial \tau$ are given by

$$
u_{\omega k_{x} k_{y}}(\tau, \xi, x, y)=\sqrt{\frac{\omega}{\pi}} \psi_{\omega k_{\perp}}(\xi) \frac{e^{i k_{x} x+i k_{y} y-i \omega \tau}}{2 \pi}
$$

where

$$
\left[-\frac{d^{2}}{d \xi^{2}}+k_{\perp}^{2} e^{2 a \xi}\right] \psi_{\omega k_{\perp}}(\xi)=\omega^{2} \psi_{\omega k_{\perp}}(\xi)
$$

and where $k_{\perp}=\sqrt{k_{x}^{2}+k_{y}^{2}}$. Requiring that $\psi_{\omega k_{\perp}}(\xi)$ decrease for $\xi \rightarrow+\infty$, we find that

$$
\psi_{\omega k_{\perp}}(\xi) \propto K_{i \omega / a}\left[\left(k_{\perp} / a\right) e^{a \xi}\right] .
$$

By the usual method of turning the normalization integral into a surface term (see, e.g., Ref. [3]), we find that the function $u_{\omega k_{x} k_{y}}$ is normalized according to Eq. (2) if, for large and negative $\xi$,

$$
\psi_{\omega k_{\perp}}(\xi) \approx-\frac{1}{\omega} \sin [\omega \xi+\alpha(\omega)] .
$$

This determines $\psi_{\omega k_{\perp}}(\xi)$ :

$$
\psi_{\omega k_{\perp}}(\xi)=\sqrt{\frac{\sinh (\pi \omega / a)}{\pi a \omega}} K_{i \omega / a}\left[\left(k_{\perp} / a\right) e^{a \xi}\right] .
$$

Consequently, we find

$$
\psi_{0 k_{\perp}}(\xi)=a^{-1} K_{0}\left[\left(k_{\perp} / a\right) e^{a \xi}\right] .
$$

We note here that $\psi_{0 k_{\perp}}(\xi) \approx-\xi+$ const for large and negative $\xi$. This can be understood as the $\omega \rightarrow 0$ limit of Eq. (10). In fact, one can directly determine the normalization factor of $\psi_{0 k_{\perp}}$ by requiring this behavior without referring to the solutions with nonzero $\omega$. We will use this method for the Schwarzschild black-hole case.

Using Eq. (8) with Eq. (11) in Eq. (5), one finds that the total response rate in the thermal bath of temperature $\beta^{-1}=a / 2 \pi$ in Rindler spacetime $R^{R}\left(k_{x}, k_{y}\right)$ is indeed equal to $R_{\mathrm{sp}}^{M}\left(k_{x}, k_{y}\right)$ given by Eq. (6). This result is interpreted as follows [3,17]: The emission of a usual finite-energy particle with transverse momentum $\left(k_{x}, k_{y}\right)$ from a uniformly accelerated source in Minkowski vacuum as described by inertial observers corresponds to either the emission or the absorption of a zero-energy Rindler particle with the same transverse momentum to or from the FDU thermal bath as described by uniformly accelerated observers. This is in agreement with Unruh and Wald's inertial interpretation of the excitation of an accelerated detector [20], and with the discussion of this problem in terms of classical radiation [21]. Although these zero-energy particles are conceptually well defined, they are not observable by the accelerated observers [3]. This is compatible with the fact that coaccelerated observers with the source do not ascribe any ordinary radiation to it $[1,2]$.

Before turning our attention to the Schwarzschild case, we will compute the integrated response rate given by the integral over the transverse momentum for later use:

$$
R^{R, \text { tot }}=R_{\mathrm{sp}}^{M, \text { tot }}=\int d k_{x} d k_{y} R_{\mathrm{sp}}^{M}\left(k_{x}, k_{y}\right)=\frac{q^{2}}{4 \pi^{2}} a .
$$

An immediate consequence of this result, and the fact that emission and absorption rates of zero-energy particles are equal, is that the total radiation rate of zero-energy particles emitted by our uniformly accelerated source is $q^{2} a / 8 \pi^{2}$.

Now, we determine the response rate of a static point source outside a Schwarzschild black hole described by a source analogous to Eq. (7) in the limit $\omega_{0} \rightarrow 0$. We use the standard Schwarzschild metric,

$$
d s^{2}=f(r) d t^{2}-f(r)^{-1} d r^{2}-r^{2}\left(d \theta^{2}+\sin ^{2} \theta d \varphi^{2}\right),
$$

where $f(r)=1-2 M / r$. The positive-frequency solutions to the massless scalar field equation in this spacetime can be written as

$$
u_{\omega l m}=\sqrt{\frac{\omega}{\pi}} \frac{\psi_{\omega l}(r)}{r} Y_{l m}(\theta, \varphi) e^{-i \omega t}
$$

Here $\psi_{\omega l}(r)$ is the solution to the differential equation

$$
\left[-f(r) \frac{d}{d r}\left(f(r) \frac{d}{d r}\right)+V_{\mathrm{eff}}(r)\right] \psi_{\omega l}(r)=\omega^{2} \psi_{\omega l}(r)
$$

where

$$
V_{\mathrm{eff}}(r)=(1-2 M / r)\left[2 M / r^{3}+l(l+1) / r^{2}\right] .
$$

For given $\omega, l$, and $m$ there are two independent and orthogonal solutions of Eq. (14). One is purely incoming from the past horizon $\mathrm{H}^{-}$and the other is purely incoming from past null infinity $\mathcal{J}^{-}$. 
In the Unruh vacuum [5], which corresponds to the physical black hole formed by gravitational collapse, a thermal flux of temperature $\beta^{-1}=1 / 8 \pi M$ comes out from $H^{-}$. In the Hartle-Hawking vacuum [22] there is an additional thermal flux coming from $\mathcal{J}^{-}$. We concentrate on the Unruh vacuum in this paper.

The regularized classical source we consider is

$$
j(x)=\frac{\sqrt{2} q f\left(r_{0}\right)^{1 / 2}}{r_{0}^{2} \sin \theta_{0}} \cos \omega_{0} t \delta\left(r-r_{0}\right) \delta\left(\theta-\theta_{0}\right) \delta\left(\varphi-\varphi_{0}\right) .
$$

This source and the source (7) have the same strength in the sense that they give the same value when integrated over the hypersurface of constant time.

Using Eq. (5) and introducing the correction factor $f\left(r_{0}\right)^{-1 / 2}$ to convert the rate per coordinate time into that per proper time, we find that the response rate per proper time of the source (15) with fixed angular momentum in the limit $\omega_{0} \rightarrow 0$ is given by

$$
R_{l m}=\frac{q^{2}}{4 \pi M r_{0}^{2}} f\left(r_{0}\right)^{1 / 2}\left|\psi_{0 l}\left(r_{0}\right)\right|^{2}\left|Y_{l m}\left(\theta_{0}, \varphi_{0}\right)\right|^{2}
$$

provided that the function $u_{\omega l m}$ in Eq. (13) is normalized according to Eq. (2). Now our task is to find the function $\psi_{0 l}$ incoming from $\mathrm{H}^{-}$and corresponding to this normalization. [Strictly speaking, we need to prove that $\psi_{\omega l}(r) \rightarrow \psi_{0 l}(r)$ as $\omega \rightarrow 0$.]

It is useful to introduce the dimensionless Wheeler tortoise coordinate $x=y+\ln (y-1)$, where $y=r / 2 M$. Equation (14) can then be rewritten as

$$
\left[-\frac{d^{2}}{d x^{2}}+(2 M)^{2} V_{\mathrm{eff}}(x)\right] \psi_{\omega l}=(2 M \omega)^{2} \psi_{\omega l}
$$

In the limit $\omega \rightarrow 0$, the incoming wave from the white-hole horizon is totally reflected towards the black-hole horizon. This implies that the Klein-Gordon normalization (2) is achieved for $u_{\omega l m}$ with $M \omega \ll 1$ if $\psi_{\omega l}$ $\approx-\omega^{-1} \sin [2 M \omega x+\alpha(\omega)]$ for large and negative $x$. Thus, in the limit $\omega \rightarrow 0$, we must normalize the solution $\psi_{0 l}$ so that

$$
\psi_{0 l} \approx-2 M x+\text { const } \quad(x<0,|x| \gg 1) .
$$

Now Eq. (14), or equivalently Eq. (17), can be solved explicitly for $\omega=0$. The general solution is [23]

$$
\psi_{0 l}(y)=C_{1} y P_{l}(2 y-1)+C_{2} y Q_{l}(2 y-1),
$$

where $P_{l}(z)$ and $Q_{l}(z)$ are Legendre functions of the first and second kinds with the branch cut $(-\infty, 1]$ for $Q_{l}(z)$. Note that $P_{l}(z) \sim z^{l}$ and $Q_{l}(z) \sim z^{-l-1}$ for large $z$ and that the solution we seek must decrease for large $y$ since the wave is totally reflected back to the horizon. From these facts and the condition (18) we find $\psi_{0 l}=4 M y Q_{l}(2 y-1)$. Substituting this in Eq. (16), we have

$$
R_{l m}=\frac{q^{2}}{\pi M} f\left(r_{0}\right)^{1 / 2}\left[Q_{l}\left(z_{0}\right)\right]^{2}\left|Y_{l m}\left(\theta_{0}, \varphi_{0}\right)\right|^{2},
$$

where $z_{0}=r_{0} / M-1$. It is possible to sum over $l$ and $m$ using the formulas

$$
\sum_{m=-l}^{l}\left|Y_{l m}(\theta, \varphi)\right|^{2}=\frac{2 l+1}{4 \pi}
$$

and

$$
\sum_{l=0}^{\infty}(2 l+1)\left[Q_{l}(z)\right]^{2}=\frac{1}{z^{2}-1}
$$

which can be obtained by squaring the formula

$$
\sum_{l=0}^{\infty} P_{l}(t) Q_{l}(z)=\frac{1}{z-t}
$$

and integrating from -1 to 1 with respect to $t$. The result is

$$
R_{\mathrm{tot}}=\sum_{l, m} R_{l m}=\frac{q^{2}}{4 \pi^{2}} a\left(r_{0}\right),
$$

where $a\left(r_{0}\right)=M f\left(r_{0}\right)^{-1 / 2} / r_{0}^{2}$ is the proper acceleration of the static source. Note that this is identical with Eq. (12) as a function of proper acceleration. This is our main result: The emission and absorption of zero-energy particles by a static source outside a Schwarzschild black hole is exactly the same as if the source were static in the Rindler wedge. In particular, the total number of zero-energy particles emitted per proper time by our source is $q^{2} a\left(r_{0}\right) / 8 \pi^{2}$.

We have not rigorously proved the validity of our approach where we directly work with the $\omega=0$ modes satisfying the normalization condition (18) instead of explicitly taking the $\omega \rightarrow 0$ limit of the modes with $\omega \neq 0$. However, the exact agreement of Eqs. (12) and (19) itself and the fact that we have reproduced with this method precisely the results of Ref. [17] serve as consistency checks of our approach. We will present elsewhere [24] a detailed analysis about how the functions $\psi_{\omega l}$ approach $\psi_{0 l}$ in the $\omega \rightarrow 0$ limit. Here we will present another consistency check using a model [25] where the effective potential $V_{\text {eff }}$ is replaced by a simpler, but similar potential (for other uses of this technique see [26])

$$
V_{\mathrm{eff}}^{(s)}(x)=l(l+1) \theta(x-1) /(2 M x)^{2}
$$

and $l \neq 0$. With this replacement, the function $\psi_{\omega l}^{(s)}$ incoming from $H^{-}$and corresponding to $\psi_{\omega l}$ can be found explicitly for any value of $\omega$, and we have

$$
\begin{aligned}
\psi_{\omega l}^{(s)}(x) & =a_{\omega l}\left(\beta_{\omega l}^{(+)} e^{i \tilde{\omega} x}+\beta_{\omega l}^{(-)} e^{-i \tilde{\omega} x}\right) \quad(x<1), \\
& =a_{\omega l} x h_{l}^{(1)}(\widetilde{\omega} x) \quad(x>1),
\end{aligned}
$$

where $\tilde{\omega}=2 M \omega$, and $h_{l}^{(1)}(x)$ is a spherical Bessel function of the third kind. Continuity of the value and the first derivative at $x=1$ gives

$$
\beta_{\omega l}^{( \pm)}=(1 / 2) e^{\mp i \tilde{\omega}}\left[(1 \mp i / \widetilde{\omega}) h_{l}^{(1)}(\widetilde{\omega}) \mp i\left(h_{l}^{(1)}\right)^{\prime}(\widetilde{\omega})\right] .
$$

The normalization condition leads to $a_{\omega l} \beta_{\omega l}^{(+)}=i / 2 \omega$ up to a phase factor. Then the mode functions with $M \omega \ll 1$ can be approximated by 


$$
\begin{aligned}
\psi_{\omega l}^{(s)}(x) & =2 M\left(1-x+l^{-1}\right)+O\left(\omega^{2}\right) \quad(x<1) \\
= & 2 M l^{-1} x^{-l}+O\left(\omega^{2}\right) \quad(x>1) .
\end{aligned}
$$

Thus, we see explicitly that the $\omega \rightarrow 0$ limit of $\psi_{\omega l}^{(s)}(x)$ is indeed $\psi_{0 l}^{(s)}(x)$ satisfying the normalization condition (18).

The importance of the zero-energy particles is conceptual rather than experimental. In some sense, they have an epistemological resemblance to virtual particles: Although unobservable, they play an important role in clarifying the physical content of some quantum phenomena. It is remarkable that our main conclusion-A scalar source has the same integrated response rate whether it is accelerated in Minkowski spacetime or static in the true gravitational field of a black hole provided the proper acceleration and underlying quantum fields are equivalent-although pertaining to the quantum realm, is in complete agreement with the classical equivalence principle. This is a nontrivial result since, in general, the equivalence principle is valid locally, while quantum mechanics deals with wave functions which are intrinsically global. Clearly, a key ingredient to reach this result was the Hawking radiation: the response rate of a static source would vanish in the absence of the Hawking effect, i.e., in the Boulware vacuum [27]. Since the Hawking effect is closely related to the absence of singularity of the quantum state on the future horizon [28,29], it is clear that the Hawk- ing effect should play a crucial role in obtaining this equivalence near the horizon. As is well known $[23,30]$, the rate of response (19) tends to the corresponding result (12) in Minkowski spacetime in the limit ${ }^{1} r_{0} \rightarrow 2 M$. However, the fact that they coincide for all $r_{0}$ was rather unexpected. It would be interesting to see if this "quantum equivalence principle" obtained here for scalar sources and Schwarzschild black holes is a special case of a more general phenomenon. The vector case for Schwarzschild black holes is currently under investigation.

We thank Bob Wald and Bernard Kay for useful discussions. We also thank Chris Fewster for helpful comments on the zero-energy limit of one-dimensional scattering theory and Mike Ryan for useful comments on the manuscript. The work of A.H. was supported in part by Schweizerischer Nationalfonds and the Tomalla Foundation. G.M. would like to acknowledge partial support from the Conselho Nacional de Desenvolvimento Científico e Tecnológico. D.S. would like to acknowledge partial support from DGAPA-UNAM Project No. IN 105496.

\footnotetext{
${ }^{1}$ We have verified that our result agrees with the standard results in Refs. $[23,30]$ in the limits $r \rightarrow 2 M$ and $r \rightarrow \infty$. The computations necessary for this comparison will be presented elsewhere [24].
}

[1] F. Rohrlich, Ann. Phys. (N.Y.) 22, 169 (1963).

[2] D. G. Boulware, Ann. Phys. (N.Y.) 124, 169 (1980).

[3] A. Higuchi, G. E. A. Matsas, and D. Sudarsky, Phys. Rev. D 45, R3308 (1992); 46, 3450 (1992).

[4] P. C. W. Davies, J. Phys. A 8, 609 (1975).

[5] W. G. Unruh, Phys. Rev. D 14, 870 (1976).

[6] S. A. Fulling, Phys. Rev. D 7, 2850 (1973).

[7] W. Rindler, Am. J. Phys. 34, 1174 (1966).

[8] S. W. Hawking, Commun. Math. Phys. 43, 199 (1975).

[9] B. S. DeWitt, in General Relativity, edited by S. W. Hawking and W. Israel (Cambridge University Press, Cambridge, England, 1979).

[10] P. G. Grove, Class. Quantum Grav. 3, 801 (1986).

[11] D. J. Raine, D. W. Sciama, and P. G. Grove, Proc. R. Soc. London A435, 205 (1991).

[12] W. G. Unruh, Phys. Rev. D 46, 3271 (1992).

[13] S. Massar, R. Parentani, and R. Brout, Class. Quantum Grav. 10, 385 (1993).

[14] F. Hinterleitner, Ann. Phys. (N.Y.) 226, 165 (1993).

[15] J. Audretsch and R. Müller, Phys. Rev. D 49, 6566 (1994).

[16] J. D. Jackson, Classical Electrodynamics, 2nd ed. (Wiley, New York, 1975).

[17] H. Ren and E. Weinberg, Phys. Rev. D 49, 6526 (1994).
[18] N. D. Birrell and P. C. W. Davies, Quantum Fields in Curved Space (Cambridge University Press, Cambridge, England, 1982).

[19] I. S. Gradshteyn and I. M. Ryzhik, Tables of Integrals, Series, and Products (Academic, New York, 1980).

[20] W. G. Unruh and R. M. Wald, Phys. Rev. D 29, 1047 (1984).

[21] A. Higuchi and G. E. A. Matsas, Phys. Rev. D 48, 689 (1993).

[22] J. B. Hartle and S. W. Hawking, Phys. Rev. D 13, 2188 (1976).

[23] P. Candelas, Phys. Rev. D 21, 2185 (1980).

[24] A. Higuchi, G. E. A. Matsas, and D. Sudarsky (in preparation).

[25] G. E. A. Matsas, D. Sudarsky, and A. Higuchi, "Bremsstrahlung by static charges outside a static black hole?', gr-qc/ 9605030 (unpublished).

[26] J. M. Aguirregabiria and C. V. Vishveshwara, Phys. Lett. A 210, 251 (1996).

[27] D. G. Boulware, Phys. Rev. D 11, 1404 (1975); 12, 350 (1975).

[28] K. Fredenhagen and R. Haag, Commun. Math. Phys. 127, 273 (1990).

[29] B. S. Kay and R. M. Wald, Phys. Rep. 207, 49 (1991).

[30] D. W. Sciama, P. Candelas, and D. Deutch, Adv. Phys. 30, 327 (1981). 